\title{
Article
}

\section{Pronounced Linewidth Narrowing of Vertical Metallic Split-Ring Resonators via Strong Coupling with Metal Surface}

\author{
Wei Du ${ }^{1,2,+}$, Youcheng Zhu ${ }^{1,+}$, Zhendong Yan ${ }^{2,3}{ }^{,}$Xiulian Xu ${ }^{1}$, Xiaoyong Xu ${ }^{1}$, Jingguo Hu ${ }^{1}$, Pinggen Cai ${ }^{4}$ \\ and Chaojun Tang ${ }^{4, *}$ \\ 1 College of Physics Science and Technology, Yangzhou University, Yangzhou 225002, China; \\ wdu@yzu.edu.cn (W.D.); yczhu_yzu@163.com (Y.Z.); xuxl@yzu.edu.cn (X.X.); xxy@yzu.edu.cn (X.X.); \\ jghu@yzu.edu.cn (J.H.) \\ 2 The National Laboratory of Solid State Microstructures, Nanjing University, Nanjing 210093, China; \\ zdyan@njfu.edu.cn \\ 3 College of Science, Nanjing Forestry University, Nanjing 210037, China \\ 4 College of Science, Zhejiang University of Technology, Hangzhou 310023, China; caippgg@zjut.edu.cn \\ * Correspondence: chaojuntang@zjut.edu.cn \\ + These authors contributed equally to this work.
}

Citation: Du, W.; Zhu, Y.; Yan, Z.; Xu, X.; Xu, X.; Hu, J.; Cai, P.; Tang, C.

Pronounced Linewidth Narrowing of Vertical Metallic Split-Ring Resonators via Strong Coupling with Metal Surface. Nanomaterials 2021, 11, 2194. https://doi.org/10.3390/ nano11092194

Academic Editor: Oleg Vitrik

Received: 1 August 2021

Accepted: 24 August 2021

Published: 26 August 2021

Publisher's Note: MDPI stays neutral with regard to jurisdictional claims in published maps and institutional affiliations.

\section{Copyright: (c) 2021 by the authors.} Licensee MDPI, Basel, Switzerland. This article is an open access article distributed under the terms and conditions of the Creative Commons Attribution (CC BY) license (https:// creativecommons.org/licenses/by/ $4.0 /)$.

\begin{abstract}
We theoretically study the plasmonic coupling between magnetic plasmon resonances (MPRs) and propagating surface plasmon polaritons (SPPs) in a three-dimensional (3D) metamaterial consisting of vertical Au split-ring resonators (VSRRs) array on Au substrate. By placing the VSRRs directly onto the Au substrate to remove the dielectric substrates effect, the interaction between MPRs of VSRRs and the SPP mode on the Au substrate can generate an ultranarrow-band hybrid mode with full width at half maximum (FWHM) of $2.2 \mathrm{~nm}$ and significantly enhanced magnetic fields, compared to that of VSRRs on dielectric substrates. Owing to the strong coupling, an anti-crossing effect similar to Rabi splitting in atomic physics is also obtained. Our proposed 3D metamaterial on a metal substrate shows high sensitivity $(S=830 \mathrm{~nm} / \mathrm{RIU})$ and figure of merit $(F O M=377)$, which could pave way for the label-free biomedical sensing.
\end{abstract}

Keywords: magnetic plasmon resonances; split-ring resonators; ultranarrow-band hybrid mode; metamaterial

\section{Introduction}

Plasmonic and metamaterial structures allow the coherent oscillations of free electrons, known as surface plasmons [1-3]. The resonant excitation of the localized surface plasmon resonances (LSPRs) and propagating surface plasmon polaritons (SPPs) concentrates light into subwavelength volumes and induces large electric field enhancements, known as "hot spots", which can be exploited in potential applications such as sensing [4,5], nonlinear optics [6,7], optical switching [8,9], photodetection [10,11], and solar energy absorbers [12,13] and related devices. Meanwhile, significant efforts have also been taken to explore nanostructures which are capable of providing localized magnetic enhancements [14-18].

Metamaterial composed of periodic arrays of sub-wavelength metallic split-ring resonators (SRRs) with the capability of enhancing magnetic field has been developed to give rise to novel electromagnetic properties and potential applications such as optical nonlinearity and magnetic biosensors [19-24]. Nevertheless, the magnetic resonance of the conventional planar SRRs usually has a relatively broad bandwidth and thus, a relatively weak enhancement of electromagnetic fields due to the fast radiation damping [25]. One effective method to enhance magnetic fields of the magnetic resonance and narrow the broad bandwidth is through coupling the MP resonance to other optical narrow-band resonance modes with high-quality factors, such as surface lattice resonances [26-28], Fabry-Perot cavity resonances [29,30], optical waveguide mode [31,32], or Tamm plasmons [33,34]. Chen et al. theoretically reported that the interactions between periodic metallic nanodisks 
and optical waveguide modes propagating in the adjacent dielectric waveguide lead to a narrow-band mixed mode with greatly enhanced magnetic fields, which can be tuned continuously by changing the array period [35].

Most of the planar SRRs are directly placed on dielectric substrates, which leads to a quite appreciable amount of electromagnetic energy spreading into the dielectric substrates and hampers the metamaterials sensing applications [20,21,36-39]. In recent years, vertical U-shaped SRRs on a dielectric substrate have been reported, in which the sensing medium can be fully spread into the free space of the hot spots of electromagnetic fields at the magnetic resonance $[40,41]$. This can be circumvented by removing the dielectric substrates and developing all-metal designs, allowing for the strong absorption of incident light through the excitation of magnetic hot spots [42-45].

In this work, we present an effective method to realize an ultranarrow-band hybrid plasmon mode with the full width at half maximum (FWHM) of $2.2 \mathrm{~nm}$ and greatly enhanced magnetic fields by plasmonic coupling between magnetic plasmon resonances (MPRs) and propagating surface plasmon polaritons (SPPs) in a three-dimensional (3D) metamaterial consisting of vertical Au split-ring resonators (VSRRs) array on Au substrate. By placing the VSRRs directly onto the Au substrate, to removing the effect of dielectric substrates, both the enhancement of the quality factor $(Q)$ and magnetic field of the VSRRs hybrid plasmon mode are up to 5 and 2 up fold compared with those of VSRRs array on dielectric substrates. Moreover, an anti-crossing phenomenon similar to Rabi splitting in atomic physics, is also observed. Our proposed 3D metamaterials on the metal substrate have high sensing performance factors $(S=830 \mathrm{~nm} / \mathrm{RIU}$ and $F O M=377)$, indicating its significant application potential in biomedical and sensing applications.

\section{Materials and Methods}

The designed 3D metamaterial is schematically depicted in Figure 1a. The array of $\mathrm{Au} \mathrm{VSRRs} \mathrm{is} \mathrm{directly} \mathrm{on} \mathrm{the} \mathrm{Au} \mathrm{substrate.} \mathrm{Figure} 1 \mathrm{~b}$ shows the magnified front view of a unit cell of the Au VSRRs structure. The structural parameter of an individual VSRR: $l_{x}=l_{z}=90 \mathrm{~nm}$ and $w=20 \mathrm{~nm}$. The periodicity $P$ along $x$ direction is set as $800 \mathrm{~nm}$. The electric field $E_{\mathrm{in}}$, magnetic field $H_{\mathrm{in}}$ and wave vector $K_{\mathrm{in}}$ of the incident light are along the $x, y$, and $z$ axes, respectively. Our proposed Au VSRRs structure is able to be achieved experimentally by the following process: Firstly, a thick Au film with a thickness of $100 \mathrm{~nm}$ is deposited on a glass substrate. Then, the Au VSRRs array is prepared by electron beam lithography with the double exposure process. We employ the commercial software package "EastFDTD, version 5.0" based on the finite-difference time-domain method to numerically simulate the plasmonic resonant behaviors of our designed Au VSRRs structure. In the $z$ axis direction and the $x$ axis direction, perfectly matched layers and periodic boundary conditions were applied, respectively. The permittivity of Au was calculated by using the Drude model [43] with the plasma frequency of $\omega_{\mathrm{p}}=1.37 \times 10^{16} \mathrm{~s}^{-1}$ and the damping constant of $\omega_{\mathrm{c}}=4.08 \times 10^{13} \mathrm{~s}^{-1}$.

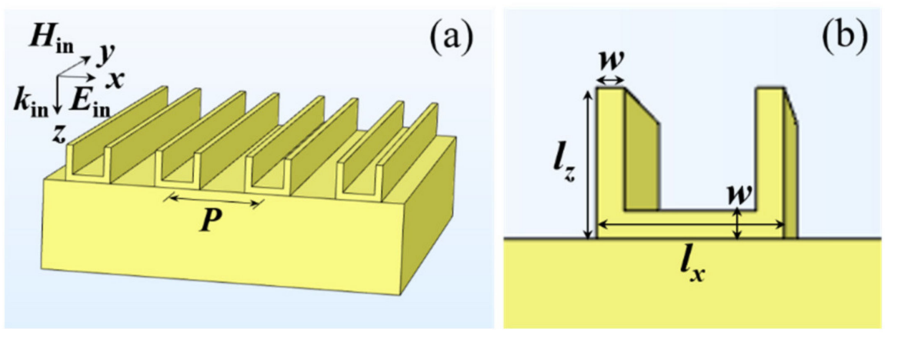

Figure 1. (a) Schematic view of the array of the Au VSRRs structure directly on the Au substrate. (b) The magnified front views of a unit cell of the Au VSRRs array.

\section{Results and Discussion}

Figure 2 shows the calculated normal-incidence reflection spectra of the designed array of Au VSRRs on Au substrate with the period $P=800 \mathrm{~nm}$. The structural parameter of 
a single VSRR is the same as shown in Figure 1. For a normal incident transverse magnetic (TM) wave, a broad reflection dip (labeled as I) centered at $696 \mathrm{~nm}$ and an ultranarrow reflection dip (labeled as II) centered at $830 \mathrm{~nm}$ are observed, which are shown by the solid red line in Figure 2, respectively. The broad reflection dip arises from the excitation of magnetic resonances in an individual Au VSRR. More importantly, the ultranarrow reflection dip with its $F W H M$ of $2.2 \mathrm{~nm}$ arises from the hybridization of propagating surface plasmon polaritons and magnetic resonances. For comparison, we also calculated the normal-incidence transmission spectra of Au VSRRs directly on silica substrate with the refractive index of 1.45 shown by the dotted blue line. The structural parameter of a single VSRR and the period are the same as those of Au VSRRs on Au substrate. There is a relatively narrow transmission dip (labeled as III) at $815 \mathrm{~nm}$ and a broad weak transmission dip on the left side of dip III at around $784 \mathrm{~nm}$ with the FWHM of $21 \mathrm{~nm}$. Such an asymmetric Fano lineshape is due to the coupling between collective diffraction mode and the magnetic resonances of Au VSRRs on a silica substrate. Nevertheless, the Fano-like transmission window is weak and the bandwidth is much broader than the reflection dip II of Au VSRRs on Au substrate because of the reduced dielectric substrate effect.

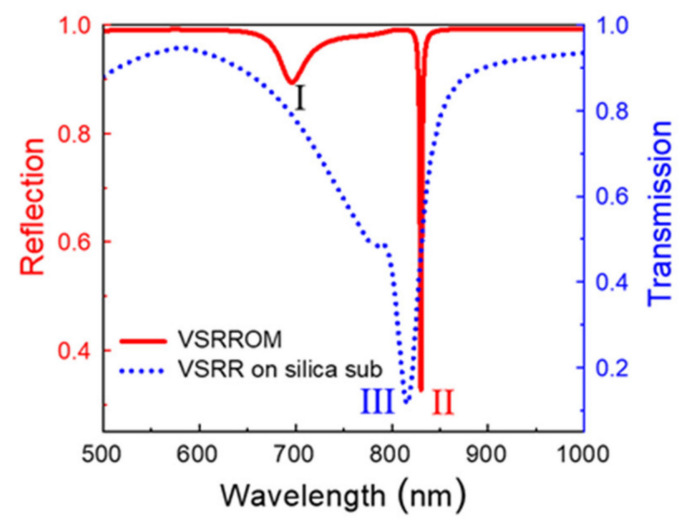

Figure 2. The calculated reflection of $\mathrm{Au}$ VSRRs on $\mathrm{Au}$ substrate and transmission of $\mathrm{Au}$ VSRRs on silica substrate. (I) denotes the MP resonance and (II) denotes the narrowband mixed mode of the Au VSRRs on Au substrate. (III) denotes the transmission dip of the SRR on a silica substrate.

Figure 3 shows the normalized electromagnetic field $\left(H / H_{\text {in }}\right.$ and $\left.E / E_{\text {in }}\right)$ distributions on the $x o z$ plane for the dip (I) and dip (II) of the Au VSRRs on Au substrate and for the dip (III) of the Au VSRRs on silica substrate, respectively. Obviously, both the magnetic field and electric field distributions of dip (II) are very similar to those of the dip (I) resonance, but the maximum magnetic field and the maximum electric field at the resonance of dip (II) are enhanced to be about 50 and 70 times of incident magnetic and electric fields, which are 2 and 2.33 times as strong as the corresponding values at the dip (III) resonance of the $\mathrm{Au}$ VSRRs on silica substrate.

In order to get a deeper insight into the nature of two hybrid modes of the Au VSRRs on Au substrate, the positions of reflection dips for different periods $P$ increased from $550 \mathrm{~nm}$ to $1050 \mathrm{~nm}$ in steps of $50 \mathrm{~nm}$ are shown by the two branches of the open circles and red lines in Figure 4. We use a coupling model to investigate the coupling effects between MP resonance and the SPPs in the proposed metamaterials by the equation [2]: $E_{ \pm}=\left(E_{\mathrm{MP}}+E_{\mathrm{SPPs}}\right) / 2 \pm \sqrt{\Delta / 2+\left(E_{\mathrm{MP}}-E_{\mathrm{SPPs}}\right)^{2} / 4}$. Here, $E_{\mathrm{MP}}$ and $E_{\mathrm{SPPs}}$ are the excitation energies of the MP resonance and the SPPs, respectively. $\Delta$ represents the value of coupling strength. The black solid line denotes the MP resonance. For our proposed structure, the incident light wavelength to excite the SPPs under normal incidence can be calculated [2]: $\lambda_{\mathrm{SPPs}}^{n}=(P / n) \sqrt{\varepsilon_{\mathrm{m}} /\left(\varepsilon_{\mathrm{m}}+1\right)}$, where $n$ is integer and $\varepsilon_{\mathrm{m}}$ is the relative permittivity of gold. The positions of reflection dips for different period $P$ can be predicted accurately, as shown by the two branches of red lines in Figure 4. At the crossing of the SPPs and the MP resonance, the reflection dips exhibit an obvious anti-crossing similar to the Rabi splitting in atomic physics, indicating the strong coupling between the SPPs and the 
MP resonance. Such strong coupling in our proposed the Au VSRRs on Au substrate is able to generate an ultranarrow hybrid mode and a large electromagnetic field enhancement at the dip II resonance.
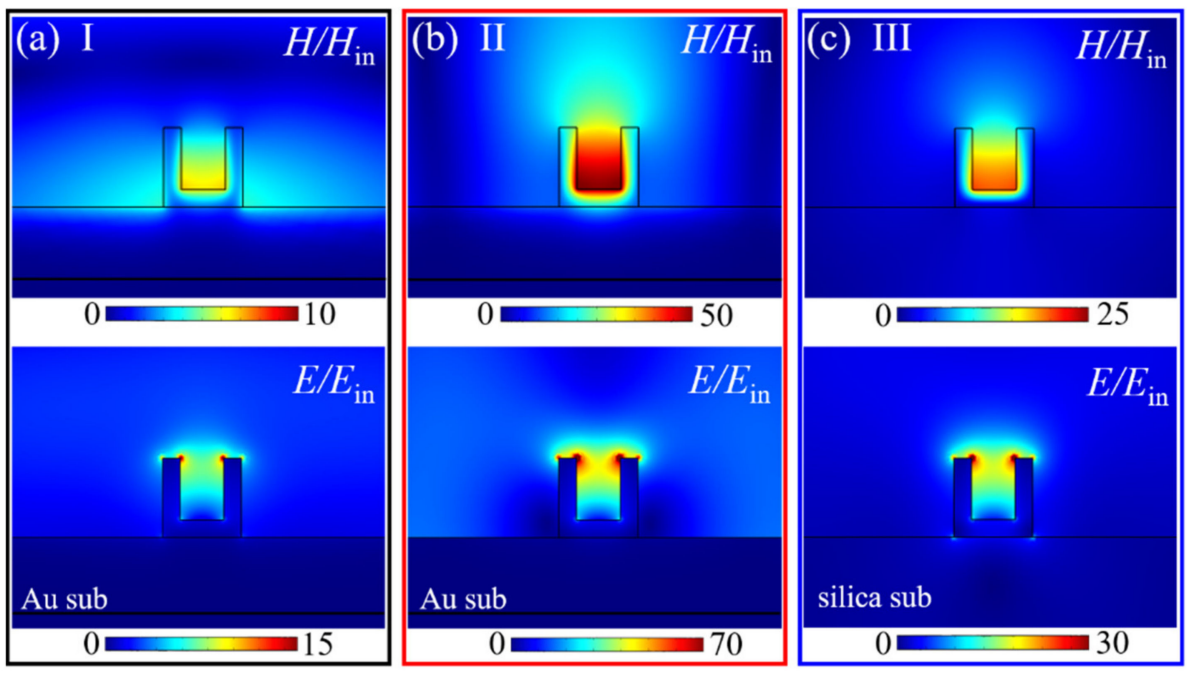

Figure 3. Normalized magnetic field $\left(H / H_{\mathrm{in}}\right)$ and electric field $\left(E / E_{\mathrm{in}}\right)$ distributions on the xoz plane for the dip I (a) and dip II (b) of the Au VSRRs on Au substrate and for the dip III (c) of the Au VSRRs on silica substrate.

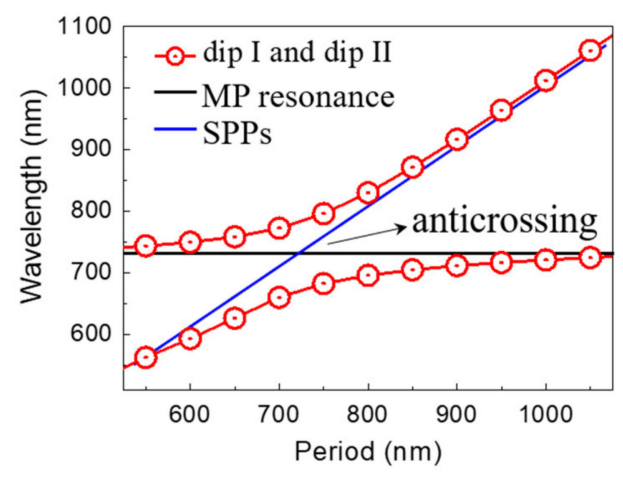

Figure 4. The dependence of the reflection dip positions (I) and (II) on the period $P$. The positions of the MP resonance (solid black line) and the SPPs (solid blue line) are also presented.

We next investigate the effects of the prong length $l_{z}$, the base rod length $l_{x}$ and the width $w$ shown in Figure $1 \mathrm{~b}$ on two hybrid modes of the Au VSRRs on Au substrate. As shown in Figure $5 \mathrm{a}$, under the condition of other fixed structural parameters $\left(l_{x}=90 \mathrm{~nm}\right.$, $w=20 \mathrm{~nm}$ and $P=800 \mathrm{~nm}$ ), when the prong length $l_{z}$ increases from $80 \mathrm{~nm}$ to $120 \mathrm{~nm}$ in steps of $10 \mathrm{~nm}$, both the reflection dips (I) and (II) show the obvious redshift. Meanwhile, the reflection intensity of the two reflection dips, (I) and (II) become weaker, and the bandwidth of the two reflection dips become broader as $l_{z}$ is increased. As shown in Figure $5 \mathrm{~b}$, when the base rod length $l_{x}$ increases from $80 \mathrm{~nm}$ to $120 \mathrm{~nm}$ in steps of $10 \mathrm{~nm}$ under the condition of other fixed structural parameters $\left(l_{z}=90 \mathrm{~nm}, w=20 \mathrm{~nm}\right.$ and $P=800 \mathrm{~nm}$ ), the reflection dips (I) shows a blueshift while the reflection dips (II) shows a redshift. Both the reflection intensity of dips (I) and dips (II) are enhanced. Figure 5c exhibits that both the reflection dips (I) and (II) show the obvious blueshift when the width $w$ increases from $10 \mathrm{~nm}$ to $30 \mathrm{~nm}$ in steps of $5 \mathrm{~nm}$ under the condition of other fixed structural parameters $\left(l_{z}=l_{x}=90 \mathrm{~nm}\right.$, and $\left.P=800 \mathrm{~nm}\right)$. Meanwhile, the reflection intensity of both the two reflection dips (I) and (II) become stronger as $w$ is increased. 

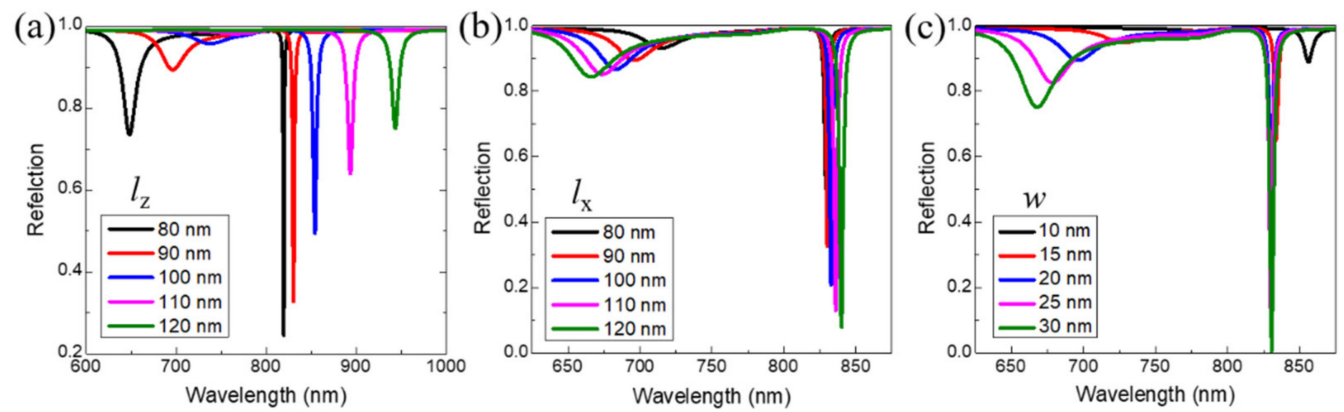

Figure 5. The calculated reflection spectra of the Au VSRRs on Au substrate (a) for different prong length $l_{z}(\mathbf{b})$ for different length of base $\operatorname{rod} l_{x}$ at normal incidence and (c) for different width $w$ of the Au split-ring resonators.

Finally, we proceed to investigate the performance of the Au VSRRs on Au substrate for optical sensing. Figure 6a shows the calculated reflection spectra of our proposed 3D metamaterials immersed in different environmental media under the TM normal incidence while keeping geometrical parameters of the Au VSRRs on Au substrate is as the same as that of in Figure 2. For the refractive index of the environmental medium increased from 1.00 to 1.04 in intervals of 0.01 , the two reflection dips (I) and (II) have obvious red-shifts. The dependence of the positions of the reflection dip II of the ultra-narrowband hybrid mode on the refractive index is shown in Figure 6b. By linearly fitting the data in Figure $6 \mathrm{~b}$, the refractive index sensitivity $(S)$ [28] of the ultra-narrowband hybrid mode of the proposed Au VSRRs structure on Au substrate is obtained to be $830 \mathrm{~nm} / \mathrm{RIU}$. The figure of merit $(F O M)$ is more meaningful to quantify the overall performance of a sensor, defined as the refractive index sensitivity divided by the resonance linewidth [28]. For the reflection dip II of the ultra-narrowband hybrid mode, its FWHM is $2.2 \mathrm{~nm}$, and the FOM is achieved to be 377 , which is enhanced up to 18 times as high as that of the reflection dip I of the MP resonance. The high FOM obtained in our proposed structure now reach nearly the highest values of the recently reported plasmonic sensors [46,47]. Such a good sensing capability of our proposed metamaterials is beneficial to highly sensitive detection of small changes of the refractive index of different environment media, which may have potential applications in biosensing.
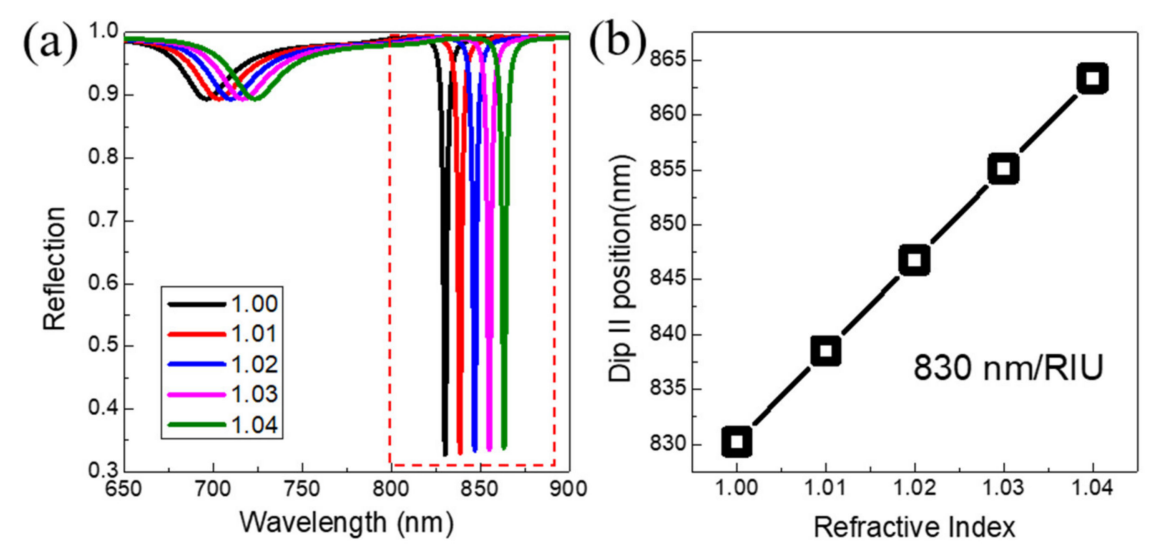

Figure 6. (a) The calculated normal-incidence reflection spectra of the Au VSRRs on Au substrate with TM polarization immersed in different environment media. (b) The resonance wavelength of the dip II extracted from (a) as a function of refractive index.

\section{Conclusions}

In conclusion, we demonstrated a powerful method to realize an ultranarrow-band hybrid plasmon mode and greatly enhanced electromagnetic fields in a 3D metamaterial composed of VSRRs array on Au substrate. By placing the VSRRs directly onto the Au 
substrate to removing the effect of dielectric substrates, both the enhancement of the quality factor $(Q)$ and magnetic field of the VSRRs hybrid plasmon mode are up to 5 and 2 up fold compared with these of VSRRs array on dielectric substrates through the strong plasmonic coupling between magnetic plasmon resonances and propagating surface plasmon polaritons. An anti-crossing phenomenon, similar to Rabi splitting in atomic physics, is also obtained. The ultra-narrowband hybrid mode of our proposed 3D metamaterials on metal substrate has an ultrahigh sensing performance factors $(S=830 \mathrm{~m} / \mathrm{RIU}$ and $F O M=377)$, indicating its significant application potential in biosensing applications.

Author Contributions: Conceptualization, J.H. and C.T.; Data curation, W.D., Y.Z. and Z.Y.; Formal analysis, X.X. (Xiulian Xu), J.H.; Funding acquisition, W.D., X.X. (Xiaoyong Xu); Investigation, W.D., Y.Z., Z.Y. and C.T.; Project administration, C.T.; Resources, X.X. (Xiulian Xu); Software, Z.Y.; Supervision, W.D. and X.X: (Xiaoyong Xu); Validation, Y.Z.; Visualization, P.C.; Writing-original draft, W.D. and Y.Z.; Writing-review \& editing, X.X. (Xiaoyong Xu), J.H. and C.T. All authors have read and agreed to the published version of the manuscript.

Funding: This research was funded by National Natural Science Foundation of China (NSFC) (51701176, 11704184, 11974303 and 12074151) and Natural Science Foundation of Zhejiang Province (LY14A040004 and LQ17C100002).

Institutional Review Board Statement: Not Applicable.

Informed Consent Statement: Not Applicable.

Data Availability Statement: The data presented in this study are available on request from the first or corresponding author.

Conflicts of Interest: The authors declare no conflict of interest.

\section{References}

1. Novotny, L.; Hecht, B. Principles of Nano-Optics; Cambridge University Press: New York, NY, USA, 2006.

2. Maier, S.A. Plasmonics: Fundamentals and Applications; Springer: New York, NY, USA, 2007.

3. Brolo, A.G. Plasmonics for future biosensors. Nat. Photonics 2012, 6, 709-713. [CrossRef]

4. Liu, Q.; Chen, X.; Fu, L.; Xie, S.; Wu, X. On-Chip Real-Time Chemical Sensors Based on Water-Immersion-Objective Pumped Whispering-Gallery-Mode Microdisk Laser. Nanomaterials 2019, 9, 479. [CrossRef]

5. Zhao, W.; Tian, X.; Fang, Z.; Xiao, S.; Qiu, M.; He, Q.; Feng, W.; Li, F.; Zhang, Y.; Zhou, L.; et al. Engineering single-molecule fluorescence with asymmetric nano-antennas. Light Sci. Appl. 2021, 10, 79. [CrossRef]

6. Xu, L.; Rahmani, M.; Kamali, K.Z.; Lamprianidis, A.; Ghirardini, L.; Sautter, J.; Camacho-Morales, R.; Chen, H.; Parry, M.; Staude, I.; et al. Boosting third-harmonic generation by a mirror-enhanced anapole resonator. Light Sci. Appl. 2018, 7, 44. [CrossRef]

7. Kim, K.H.; Rim, W.S. Strongly resonant metasurfaces supported by reflective substrates for highly efficient second- and highharmonic generations with ultralow pump intensity. Phys. Chem. Chem. Phys. 2019, 21, 19076-19082. [CrossRef]

8. Galanty, M.; Shavit, O.; Weissman, A.; Aharon, H.; Gachet, D.; Segal, E.; Salomon, A. Second harmonic generation hotspot on a centrosymmetric smooth silver surface. Light Sci. Appl. 2018, 7, 49. [CrossRef]

9. Chang, W.S.; Lassiter, J.B.; Swanglap, P.; Sobhani, H.; Khatua, S.; Nordlander, P.; Halas, N.J.; Link, S.A. A plasmonic fano switch. Nano Lett. 2012, 12, 4977. [CrossRef]

10. Lu, H.; Liu, X.; Wang, L.; Gong, Y.; Mao, D. Ultrafast all-optical switching in nanoplasmonic waveguide with Kerr nonlinear resonator. Opt. Express 2011, 19, 2910-2915. [CrossRef]

11. Knight, M.W.; Sobhani, H.; Nordlander, P.; Halas, N.J. Photodetection with active optical antennas. Science 2011, 332, 702-704. [CrossRef]

12. Miao, X.; Yan, L.; Wu, Y.; Liu, P.Q. High-sensitivity nanophotonic sensors with passive trapping of analyte molecules in hot spots. Light Sci. Appl. 2021, 10, 5. [CrossRef]

13. Feng, H.; Li, X.; Wang, M.; Xia, F.; Zhang, K.; Kong, W.; Dong, L.; Yun, M. Ultrabroadband metamaterial absorbers from ultraviolet to near-infrared based on multiple resonances for harvesting solar energy. Opt. Express 2021, 29, 6000-6010. [CrossRef]

14. Chen, Y.; Liu, C.; Situ, Y.; Liu, J.; Huang, H. Enhancing Thermal Conductivity and Photo-Driven Thermal Energy Charging/Discharging Rate of Annealed CMK-3 Based Phase Change Material. Nanomaterials 2019, 9, 364. [CrossRef] [PubMed]

15. Smith, D.R.; Padilla, W.J.; Vier, D.C.; Nemat-Nasser, S.C.; Schultz, S. Composite medium with simultaneously negative permeability and permittivity. Phys. Rev. Lett. 2000, 84, 4184-4187. [CrossRef]

16. López-Ortega, A.; Zapata-Herrera, M.; Maccaferri, N.; Pancaldi, M.; Garcia, M.; Chuvilin, A.; Vavassori, P. Enhanced magnetic modulation of light polarization exploiting hybridization with multipolar dark plasmons in magnetoplasmonic nanocavities. Light Sci. Appl. 2020, 9, 49. [CrossRef] 
17. Klein, M.W.; Enkrich, C.; Wegener, M.; Linden, S. Second-harmonic generation from magnetic metamaterials. Science 2006, 313, 502-504. [CrossRef] [PubMed]

18. Yao, J.; Wu, Y.; Liu, J.; Liu, N.; Cai, G.; Liu, Q.H. Enhanced optical bistability by coupling effects in magnetic metamaterials. J. Lightwave Technol. 2019, 37, 5814-5820. [CrossRef]

19. Liu, B.; Tang, C.; Chen, J.; Xie, N.; Yuan, J.; Tang, H.; Zhu, X. Metal-substrate-enhanced magnetic dipole resonance in metamaterials for high-performance refractive index sensing. Opt. Mater. Express 2018, 8, 2008-2016. [CrossRef]

20. Lee, H.J.; Yook, J.G. Biosensing using split-ring resonators at microwave regime. Appl. Phys. Lett. 2008, 92, 25. [CrossRef]

21. Lee, H.J.; Lee, H.S.; Yoo, K.H.; Yook, J.G. DNA sensing using split-ring resonator alone at microwave regime. J. Appl. Phys. 2010, 108, 014908. [CrossRef]

22. Shadrivov, I.V.; Kozyrev, A.B.; van der Weide, D.W.; Kivshar, Y.S. Nonlinear magnetic metamaterials. Opt. Express 2008, 16, 20266-20271. [CrossRef]

23. Huang, D.; Rose, A.; Poutrina, E.; Larouche, S.; Smith, D.R. Wave mixing in nonlinear magnetic metacrystal. Appl. Phys. Lett. 2011, 98, 204102. [CrossRef]

24. Pol, L.; Eckstein, C.; Acosta, L.K.; Xifré-Pérez, E.; Ferré-Borrull, J.; Marsal, L.F. Real-Time Monitoring of Biotinylated Molecules Detection Dynamics in Nanoporous Anodic Alumina for Bio-Sensing. Nanomaterials 2019, 9, 478. [CrossRef]

25. Tobing, L.Y.M.; Tjahjana, L.; Zhang, D.H.; Zhang, Q.; Xiong, Q.H. Deep subwavelength fourfold rotationally symmetric split-ringresonator metamaterials for highly sensitive and robust biosensing platform. Sci. Rep. 2013, 3, 2437. [CrossRef]

26. Fang, M.; Shen, N.H.; Sha, W.E.I.; Huang, Z.; Koschny, T.; Soukoulis, C.M. Nonlinearity in the dark: Broadband terahertz generation with extremely high efficiency. Phys. Rev. Lett. 2019, 122, 027401. [CrossRef]

27. Chen, J.; Mao, P.; Xu, R.; Tang, C.; Liu, Y.; Wang, Q.; Zhang, L. Strategy for realizing magnetic field enhancement based on diffraction coupling of magnetic plasmon resonances in embedded metamaterials. Opt. Express 2015, 23, 16238-16245. [CrossRef]

28. Tang, C.J.; Zhan, P.; Cao, Z.S.; Pan, J.; Chen, Z.; Wang, Z.L. Magnetic field enhancement at optical frequencies through diffraction coupling of magnetic plasmon resonances in metamaterials. Phys. Rev. B 2011, 83, 041402. [CrossRef]

29. Yan, Z.D.; Zhu, Q.; Wan, M.J.; Lu, X.; Ting, X.; Tang, C.J.; Yu, L. Graphene ultraviolet ultrahigh-Q perfect absorption for nanoscale optical sensing. Opt. Express 2020, 28, 6095-6101. [CrossRef]

30. Yang, Z.; Chen, Y.; Zhou, Y.; Wang, Y.; Dai, P.; Zhu, X.; Duan, H. Microscopic interference full-color printing using grayscalepatterned Fabry-Perot resonance cavities Microscopic interference full-color printing using grayscale-patterned Fabry-Perot resonance cavities. Adv. Opt. Mater. 2017, 5, 1700029. [CrossRef]

31. Wang, S.; Chen, W. A Large-Area and Nanoscale Graphene Oxide Diaphragm-Based Extrinsic Fiber-Optic Fabry-Perot Acoustic Sensor Applied for Partial Discharge Detection in Air. Nanomaterials 2020, 10, 2312. [CrossRef]

32. White, J.M.; Heidrich, P.F. Optical waveguide refractive index profiles determined from measurement of mode indices: A simple analysis. Appl. Opt. 1976, 15, 151-155. [CrossRef]

33. Priambodo, P.S.; Maldonado, T.A.; Magnusson, R. Fabrication and characterization of high-quality waveguide-mode resonant optical filters. Appl. Phys. Lett. 2003, 83, 3248-3250. [CrossRef]

34. Wang, X.; Jiang, X.; You, Q.; Guo, J.; Dai, X.; Xiang, Y. Tunable and multichannel terahertz perfect absorber due to Tamm surface plasmons with graphene. Photonics Res. 2017, 5, 536-542. [CrossRef]

35. Auguié, B.; Bruchhausen, A.; Fainstein, A. Critical coupling to Tamm plasmons. J. Opt. 2015, 17, 035003. [CrossRef]

36. Chen, J.; Nie, H.; Zha, T.; Mao, P.; Tang, C.; Shen, X.; Park, G. Optical magnetic field enhancement by strong coupling in metamaterials. J. Lightwave Technol. 2018, 36, 2791-2795. [CrossRef]

37. Dmitriev, A.; Hagglund, C.; Chen, S.; Fredriksson, H.; Pakizeh, T.; Kall, M.; Sutherland, D.S. Enhanced nanoplasmonic optical sensors with reduced substrate effect. Nano Lett. 2008, 8, 3893-3898. [CrossRef] [PubMed]

38. Wu, P.C.; Sun, G.; Chen, W.T.; Yang, K.Y.; Huang, Y.W.; Chen, Y.H.; Huang, H.L.; Hsu, W.L.; Chiang, H.P.; Tsai, D.P. Vertical split-ring resonator based nanoplasmonic sensor. Appl. Phys. Lett. 2014, 105, 033105. [CrossRef]

39. Wu, P.C.; Hsu, W.L.; Chen, W.T.; Huang, Y.W.; Liao, C.Y.; Liu, A.Q.; Zheludev, N.I.; Sun, G.; Tsai, D.P. Plasmon coupling in vertical split-ring resonator metamolecules. Sci. Rep. 2015, 5, 9726. [CrossRef]

40. Wu, P.C.; Papasimakis, N.; Tsai, D.P. Self-affine graphene metasurfaces for tunable broadband absorption. Phys. Rev. Appl. 2016, 6, 044019. [CrossRef]

41. Ma, Q.; Dai, J.; Luo, A.; Hong, W. Numerical and Theoretical Study of Tunable Plasmonically Induced Transparency Effect Based on Bright-Dark Mode Coupling in Graphene Metasurface. Nanomaterials 2020, 10, 232. [CrossRef]

42. Wang, X.; Meng, H.; Deng, S.; Lao, C.; Wei, Z.; Wang, F.; Tan, C.; Huang, X. Hybrid Metal Graphene-Based Tunable PlasmonInduced Transparency in Terahertz Metasurface. Nanomaterials 2019, 9, 385. [CrossRef]

43. Sobhani, A.; Manjavacas, A.; Cao, Y.; McClain, M.J.; García de Abajo, F.J.; Nordlander, P.; Halas, N.J. Pronounced linewidth narrowing of an aluminum nanoparticle plasmon resonance by interaction with an aluminum metallic film. Nano Lett. 2015, 15, 6946-6951. [CrossRef] [PubMed]

44. Liu, N.; Liu, H.; Zhu, S.; Giessen, H. Stereometamaterials. Nat. Photonics 2009, 3, 157-162. [CrossRef]

45. Symonds, C.; Bonnand, C.; Plenet, J.C.; Bréhier, A.; Parashkov, R.; Lauret, J.S.; Deleporte, E.; Bellessa, J. Particularities of surface plasmon-exciton strong coupling with large Rabi splitting. New J. Phys. 2008, 10, 065017. [CrossRef] 
46. Mayer, K.M.; Hafner, J.H. Localized surface plasmon resonance sensors. Chem. Rev. 2011, 111, 3828-3857. [CrossRef]

47. Chen, J.; Qi, S.; Hong, X.; Gu, P.; Wei, R.; Tang, C.; Huang, Y.; Zhao, C. Highly sensitive 3D metamaterial sensor based on diffraction coupling of magnetic plasmon resonances. Results Phys. 2019, 15, 102791. [CrossRef] 